\title{
Implante de titanio sometido a carga inmediata. Análisis histológico del tejido óseo y evaluación cualitativa y cuantitativa de la superficie del implante
}

\author{
Corina M.* \\ Savoldi E.*
}

\begin{abstract}
Corica M, Savoldi E; Implante de titanio sometido a carga inmediata. Análisis histológico del tejido óseo y evaluación cualitativa y cuantitativa de la superficie del implante, 2000; 12
\end{abstract}

\begin{abstract}
RESUMEN
Sabemos muy bien que la estabilidad de un implante endo-óseo está ligada a múltiples factores: integridad del sitio del implante, cantidad y calidad del hueso, microtopografía del implante y tiempo de recuperación clínica que permita una buena mineralización de la zona del hueso neoformado que rodea el implante.

No todos los autores están de acuerdo en que el implante permanezca completamente sumergido durante la fase de recuperación. Algunos creen que la carga inmediata sobre los implantes acelera la transformación del hueso indiferenciado en hueso laminar denso, el cual resiste mejor a las fuerzas de la masticación.

En el caso clínico que expondremos a continuación, el implante con cuello transmucoso colocado en el sitio del incisivo central izquierdo superior fue expuesto a trauma tres meses después de ser insertado.

El caso nos permitió examinar la superficie del implante con microscopio electrónico explorador (SEM) el cual mostró células similares a osteoblastos. Estos resultados luego fueron confirmados por la biopsia histológica, la cual bajo el microscopio óptico mostró señas de remodelación ósea, es decir indicativas de que el implante se había estabilizado durante el período de curación.
\end{abstract}

\section{PALABRAS CLAVE:}

Implantología - carga inmediata - integración ósea

\section{INTRODUCCIÓN}

Es ya una convicción común que, en un contacto directo hueso-implante es obligatorio que este último permanezca inmóvil durante el período de curación. Se ha reconocido la importancia de la preparación quirúrgica del sitio del implante y de la integridad del mismo entendida como cantidad y calidad de hueso cortical y hueso medular cuya densidad es diferente entre el maxilar y la mandíbula, siendo mayor en esta última.

Además la microtopografía del implante, es decir la geometría y las características de la superficie del implante son muy significativas en la fase de inser- ción para consentir una retención inmediata entre hueso e implante. Si el implante es sometido a microesfuerzos durante el período de curación sea a través de una prótesis provisional sobre la que se aplican cargas excesivas, sea porque el mismo paciente produce trauma en el implante, entonces se puede perjudicar el contacto sólido del implante (estabilidad primaria) con la superficie ósea del sitio receptor dando como resultado la movilidad y el fracaso, debido a la formación de un espeso tejido conjuntivo colágeno en la superficie interfacial.

En el proceso de integración ósea participan tres tipos de hueso: hueso denso, hueso laminar y hueso compuesto o mixto. 
El hueso denso se forma rápidamente en respuesta al estímulo y es responsable de la estabilización del implante endo-óseo en la fase de curación inicial. Tiene una baja densidad de minerales y una orientación causal de las trabéculas. Aunque es capaz de estabilizar un implante no sometido a carga, no logra resistir bajo carga funcional.

El hueso laminar se forma más lentamente que el hueso compacto y presenta una matriz organizada altamente mineralizada, de hueso cortical maduro y hueso trabecular que constituye el sostén principal de la carga que se ejerce sobre el conjunto huesoimplante. El hueso compuesto resulta de hueso laminar que se ha depositado en la matriz ósea densa y representa la última etapa en la estabilización del implante endo-óseo durante su integración. La interfase del hueso de sostén que sigue a la inserción de un implante requiere aproximadamente 12 meses (19), de los cuales los primeros cuatro meses para la regeneración sin carga, caracterizada ya en la primera semana por el depósito del $70 \%$ de los minerales en el hueso maduro vital y los restantes ocho meses por la fase de maduración.

Según la mayor parte de los autores, la remineralización es importante porque contribuye a la dureza y fuerza del hueso laminar y la fuerza total del mismo que sostiene a un implante endo-óseo no se puede conseguir antes de que se complete la mineralización secundaria del hueso neoformado.

El requisito indispensable de que el implante permanezca sumergido durante la fase de curación no es aceptado universalmente. En efecto, según algunos autores (4-22-26) la carga inmediata sobre los implantes favorece la rapidez de transformación del hueso indiferenciado en hueso laminar denso capaz de resistir a las fuerzas masticatorias.

La técnica quirúrgica en un solo tiempo ofrece las ventajas del mantenimiento en amplitud en la mucosa queratinizada, mientras que los márgenes de la herida se adaptan bien al cuello del implante emergente, lo que permite la aplicación de los pilares (abutment) en condiciones de excelente visibilidad (4).

\section{MATERIALES Y MÉTODOS}

El objetivo de este trabajo es el de analizar desde el punto de vista histomorfométrico la calidad estructural del hueso neoformado en contacto con un implante sometido a carga inmediata, además de evaluar la superficie del implante bajo el aspecto cualitativo y cuantitativo, al microscopio electrónico explorador (SEM).

\section{Caso clínico}

Sujeto de 30 años con el diente de la posición 11, reimplantado tres años antes, en estado de RIZÓLISIS y notablemente móvil; se decidió la extracción del elemento dental residual. Sólo después de cuatro semanas se procedió a la inserción de un implante de titanio grado 2 de superficie arenada de 4 milímetros de diámetro, 13 milímetros de largo, con el cuello de 2 milímetros de altura, asociado a un injerto de hueso autólogo obtenido de la regularización del cráter alveolar. Considerado el sitio anatómico y con el consentimiento total del paciente se pensó en apoyar sobre el cuello transmucoso una corona provisional de resina cocida con dos aletas de extensión que se fijó con material compuesto por el lado del paladar de cada uno de los dos dientes adyacentes. Después de unas 12 semanas de la operación el paciente se volvió a presentar al consultorio después de un traumatismo accidental que le provocó la fractura conminuta de la pared ósea vestibular correspondiente a nuestro implante, perjudicando su estabilidad. Aprovechamos dicha oportunidad para someter a un análisis histológico el tejido que rodeaba el implante.

La muestra fue fijada en formalina al 10\%, deshidratada en alcohol al 95\% e incluida en polimetilmetacrilato; entonces utilizando un micrótomo se prepararon secciones de $7 \mathrm{~mm}$ (milimicrones) de espesor para poner en evidencia la variedad de las condiciones del tejido en la interfase.

Antes de pasar a la observación in vitro con el microscopio óptico de luz polarizada, se procedió a colorear según los componentes tisulares que se habian de evidenciar.

\section{RESULTADOS}

A la observación con el microscopio óptico las diversas muestras de la biopsia revelan que se trata de tejido óseo compacto y de armaduras óseas mineralizadas con señales de neoformación y de reabsorción (Fig. 1-2) bien evidenciada en la ampliación mayor a 200 x y teñidas con el Tricromo de Goldner.

El análisis histológico al microscopio óptico con luz polarizada del hueso adherido a la superficie del implante puso en evidencia una congruencia anatómica, o mejor dicho, un íntimo contacto entre el hueso y el implante cargado inmediatamente (Fig. 3), con escasa cantidad de tejido conjuntivo y, a nivel de la espira, la tendencia de las células óseas en curso de maduración es a organizar una estructura Haversiana nueva alrededor del implante. 


\section{Azul de toluidina - Fucsina básica}

caracterizada por el hecho de que los colorantes tisulares como el azul y el rojo a la observación con el microscopio óptico adquieren una tonalidad diversa denominada coloración metacromática.

\section{Tricromo de Goldner}

permite una buena observación de los núcleos de las células coloreadas de rojo, mientras que el tejido conjuntivo se presenta en verde y aquél no mineralizado en rojo anaranjado.

\section{Hematoxilina y Eosina}

es el método empleado comúnmente. Pone en evidencia los núcleos en azul y el citoplasma en rosado.

TABLA 1 - Coloración histológica

La confirmación de su naturaleza nos la otorga el microanálisis efectuado con el método Edax-Econ. Por este motivo introdujimos la muestra de titanio que se analizó con una cámara de ultravacío donde el titanio es golpeado por electrones acelerados bajo una adecuada diferencia de potencial de 1 a $30 \mathrm{Kev}(\mathrm{kW})$ de modo que sus átomos de superficie emitan rayos $\mathrm{X}$ cuya intensidad y energía son características de cada elemento presente y por lo tanto traducibles gráficamente bajo forma de picos que en su conjunto constituyen el espectro.

En el caso examinado, el histograma muestra en la substancia matriz la presencia de calcio y fósforo en proporciones casi iguales (ver tabla II).

\section{DISCUSIÓN Y CONCLUSIONES}

Las secciones seriadas no descalcificadas de la muestra bióptica a lo largo del plano longitudinal mesiodistal, gracias a los diversos métodos de coloración permiten evidenciar bien, con el microscopio óptico de luz polarizada, el dinamismo óseo en la zona de obtención de la muestra, y por consiguiente la actividad osteogénica.

Además de la densidad ósea alrededor del alvéolo, en nuestro caso hueso de tipo 1 según Misch, uno de los factores que entra en juego para favorecer el éxito

\begin{tabular}{|c|c|c|c|}
\hline $\begin{array}{l}\text { TABLA } 2 \\
\text { LIST: }\end{array}$ & LABEL $=$ VITE & 18-MAR-96 $\quad 00: 48: 50$ & 60.004 LIVE SECONDS \\
\hline ELEMENTS & INTENSITY (CPS) & BACKGROUND (CPS) & P/B RATIO \\
\hline NA K & 10.733 & 7.483 & 1.434 \\
\hline $\mathrm{SI} \mathrm{K}$ & 7.833 & 24.498 & 0.312 \\
\hline PK & 17.266 & 27.498 & 0.628 \\
\hline SK & 30.381 & 32.181 & 0.944 \\
\hline $\mathrm{CLK}$ & 9.483 & 33.231 & 0.255 \\
\hline $\mathrm{CAK}$ & 11.333 & 34.631 & 0.327 \\
\hline TI K & 3.017 .799 & 26.248 & 114.971 \\
\hline ZN K & 35.599 & 13.649 & 2.608 \\
\hline
\end{tabular}

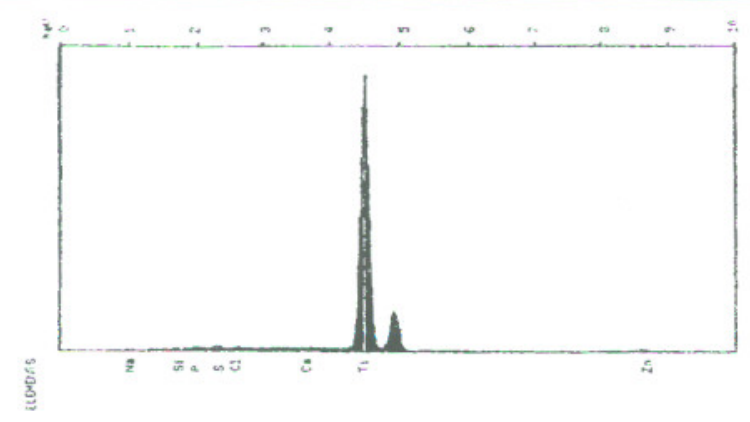

ITIS CASTELLI BRESCIA 25-MAR-96

FILENAME: AGOCO1, BSPDATE: 18-MAR-96

LABEL: VITE

RANGE: 0 - 10

EV/CH: 10

FS: 2433

NOTE: 
del implante, intercalado entre dientes naturales y puesto bajo carga de inmediatol8-20-25, pareciera estar también en la integridad del sitio anatómico y en el caso indicado se trataba de incisivo central izquierdo superior expuesto a esfuerzos poco significativos.

Según la escuela de Branemark (1), cuando en la superficie de un implante cargado funcionalmente y observado al microscopio óptico se demuestra la presencia de tejido mineralizado a lo largo de una parte significativa de su perímetro externo, éste se clasifica como osteointegrado. Además los últimos datos de la investigación científica (12) han evidenciado que la inserción del implante después de al menos dos semanas desde la preparación de su alvéolo o lecho quirúrgico ofrece una formación ósea extensa precoz y menor presencia de tejido fibroso a su alrededor.

En el caso señalado, el implante estaba rodeado de hueso vital compacto con áreas de remodelación, superficies libres de hueso y señas de reabsorción.

Estos resultados demuestran que el implante estaba en condición estable durante el período de curación y por esto había entrado en contacto directo con el hueso, a pesar de la poco significativa presencia de tejido conjuntivo. Aunque estimulante, dicho resultado quiere significar cómo un implante insertado en una determinada área anatómica, intercalado entre dientes frontales, puesto bajo carga de inmediato y controlado puede enfrentar el proceso de integración ósea.

\section{SUMMARY}

It is well known that the stability of an endosseous implant is linked to several factors: integrity of the implant site, size and quality of bone, microtopography of the implant element and time of clinical recovery allowing for good mineralization of the new formed perimplantar bone.

Not all authors agree that the implant is fully submerged during recovery.

Some believe that the immediate load on implants quickens the transformation of the indifferenziated bone, which is therefore more capable of bearing masticatory loads.

In the clinical case shown, the implant with transmucous neck placed in the site of the upper left central tooth was exposed to trauma 3 months after the operation.

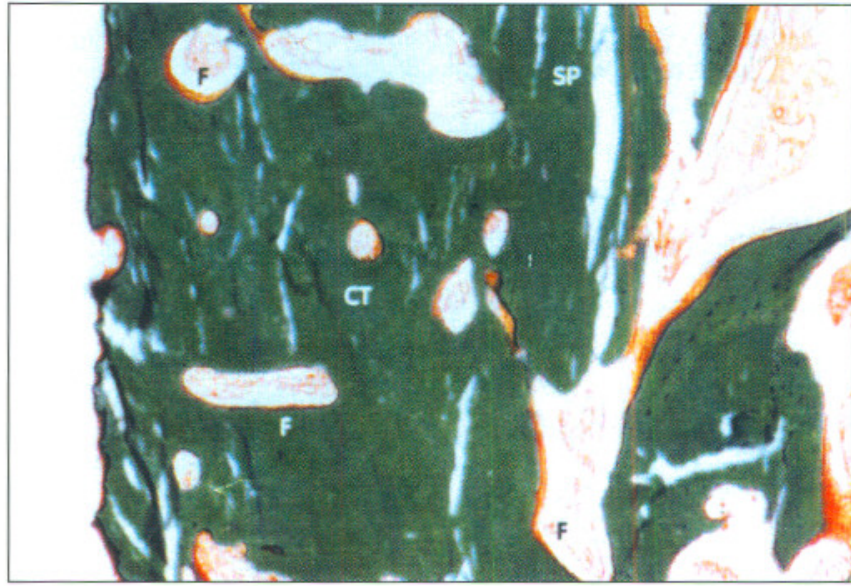

Fig. 1: Análisis muestra histológica circundante al implante con ampliación $62.5 \mathrm{X}$

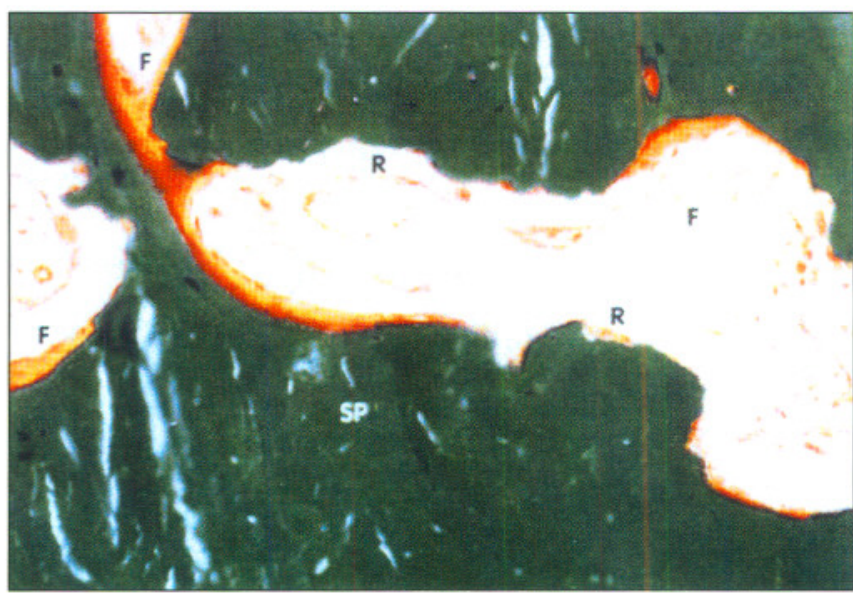

Fig. 2. Ampliación x 200. Esta muestra resulta de tejido óseo compacto $(C T)$ por trabéculas óseas (SA) con áreas de formación $(F)$ $y$ de reabsorción $(R)$.

La coloración empleada es al triocomo de Goldner

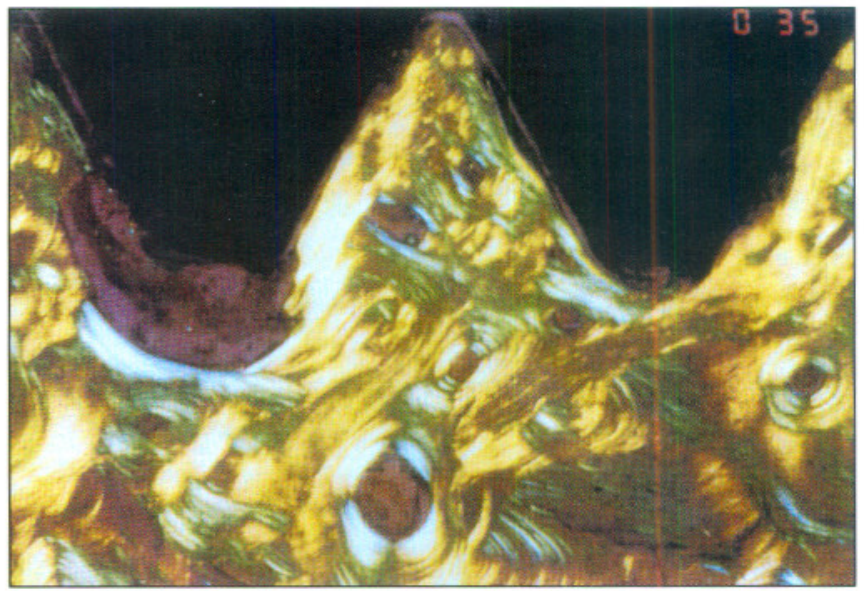

Fig. 3. Vista al microscopio óptico con luz polarizada del hueso alrededor de implante cargado inmediatamente. Esta ampliación $400 \mathrm{X} \mathrm{mm}$ pone en evidencia una presencia osteónica gracias a la coloración con azul de toluidina = fucsina básica 
The case allowed us to examine the implant surface by scan electron microscopy which showed cells similar to osteoblasts. These results were later confirmed by the histological biopsy, which under the optic microscope showed signs of osseous remodelling, thus suggesting that the implant was stabilised during recovery.

\section{KEY WORDS:}

Implantology - immediate loading - osseointegration

\section{CORRESPONDENCIA}

\section{Corica Marino}

Vía Risorgimento, 40

I - 25127 BRESCIA

Tel.: 0039-030-304363

\section{BIBLIOGRAFÍA}

1. Albrektsson T., Sennerby L. : State of the art in oral implants. J. Clin. Periodontol. 18:474-481, 1991.

2. Albrektsson T., Johansson C.: Quantified bone tissue reactions to various metallic materials with reference to the so called osseointegration concept. In : DAVIES JE (Ed.) the bone biomaterial interface. Toronto Univ. Of Toronto 357-363, 1991.

3. Benn D.K. : A review of the reliability of radiographic measurements in estimating alveolar bone changes. J. Clin. Periodontol. 17:14-21, 1990.

4. Buser D, Weber H., Lang N.P. : Tissue integration of one stage ITI implants. In : Laney W.R., Tolman D.E. (eds): Tissue integration in Oral Orthoped. and Maxillofacial Reconstruction, Chicago, Quintessence 8:362-268, 1992.

5. Brunette DM., Raktay J., Chehroudi B. : Behaviour of osteoblasts on micromachined surfaces. In : Davies J.E. (ed) The bone-biomaterial interface, Toronto, Univ. of Toronto 170-179, 1991.

6. Caudill R.F., Meffert R.M. : Histologic analysis of the osseointegration of endosseous implants in simulated extraction sockets with and without e PTFE barriers. Part I Preliminary findings. Int. J. Periodont. Rest. Dent. 11:207-216, 1991.

7. Donath K. : Preparation of histologic sections. Norderstedt, Germany, EX AKT Kulzer 1987.
8. Ericsson I., Randow K., Glantz P.O., Lindhe J., Nilner K. : Some clinical and radiographical features of submerged and non submerged implants. Clin. Oral Res. 5:185-189, 1994.

9. Goffredsen K., Hjørting - Hansen E. : Histologic and hystomorphometric evaluation of submerged and non submerged titanium implants. In : Laney W.R., Tohman D.E. (Eds) Tissue integration in Oral Orthopedic and Maxillofacial Reconstruction. Chicago : Quintessence 37-40, 1992.

10. Henry P., Rosenberg J. : Single stage surgery for rehabilitation of the endentulous mandible: Preliminary results. The implant Report Vol. 6, Nœ9, 1994.

11. Johansson C.B., Albrektsson T. : A removal torque and histomorphometric study of commercially pure niobium and titanium implants in rabbit bone. Clinical Oral Implants Research 2: 24-29, 1991.

12. Knox R., Caudill R., Meffert R : Etude histologique d'implants endosseux dans les defauts extractionells chirurgicalement arées. Revue internationale de Parodontie et dentisterie restauratrice, Vol. 11, nळ 5, 1991.

13. Listgarten M.A., Buser D., Steinman S.G., Donath K., Lang N.P., Weber H.P. : Light and transmission electron microscopy of the intact interfaces between non submerged titanium coated epoxy resin implants and bone and gingiva. J. Dental Res. 71:364-371, 1991.

14. Lozada J.L., James R.A., Boscovic M. et al: Surgical repair of perimplant defects. J. Oral Implantol. 16: 42$46,1990$.

15. Misch C.E. : Density of bone: effect on treatment plans, surgical approache, healing, and progressive bone loading. Int. J. Oral Implantol. 6:23-31, 1990.

16. Misch C.E. : Progressive loading of bone with implant prostheses. J. Dent Symposia 1:50-53, 1993.

17. Morris H.F., Ocmi S. : The influence of implant design, application, and site on clinical performance and crestal bone: a multicenter, multidisciplinary clinical. Study Implant Dent. 1:49-55, 1992.

18. Ogiso M., Tabata T., Lee R..R., Borgese D. : Enhances implant-bone binding: a comparison with the conventional method. Int. J. Oral Maxillofac-Implants Jul-Aug. $10(4): 415-420,1995$.

19. Piattelli A. : Early and immediate loading in oral implantoprosthesis, possibility and limits. Roma 2nd World Congress of Osseointegration 48, 1998. 
20. Roberts W.E. : Fundamental principles of bone physiology, metabolism and loading. In : Naert I., Van Steenberghe D., Worthington P., eds. Osseointegration in Oral Rehabilitation. Carol Stream, IL Quintessence Publishing Co. 157-170, 1993.

21. Ruggeri A., Franchi M., Marini N., Trisi P., Piattelli A. : Supracrestal circular collagen fiber network around osseointegrated nonsubmerged titanium implants. Clin. Oral Implants Res 3: 169-175, 1992.

22. Tardieu P. : Immediate placement and loading of implants into fresh extraction sokets. Annual Congress of the American Accademy of Periodontology, New Orleans October, 1996.

23. Uhthoff H.K. : Mechanical factors influencing the holding power of screws in compact bone. J. Bone Joint Surg. (Br.) 55:663-639, 1973.
24. Weber H.P., Buser D., Fiorellino J.P., Williams R.C. : Radiographic evaluation of crestal bone levels adiacent to non submerged titanium implants. Clin. Oral Impl. Res. 3:181-188, 1992.

25. Webirt M., Goldberg P. : L'implant immediat: preservation et regeneration osseuse. Revue Internationale de Parodontologie e dentisterie restauratrice. Vol. 12, nœ3, 1992.

26. Weiss C.M. : A comparative analysis of fibrosteal and osteal integration and others variables that affect long term bone maintenance around dental implants. The Journal of Oral Implantology Vol. XII, Nœ3, 467-486, 1987.

27. Wilke H.J., Claes L., Steinemann S. : The influence of various titanium surfaces on the interface shear strenght between implants and bone in : Clinical materials. Heinake G., Soltesz V. and Lee AJC, Eds. Elsevier science publishers, Amsterdam 1990. 